\title{
VALVULAR (EXTERNAL HINGE) LEAKAGE OF THE CARBOMEDICS MITRAL VALVE
}

Kazuhiro Hashimoto, MD, ${ }^{a}$ Tatsuta Arai, MD, ${ }^{a}$ Nariyuki Takeuchi, MD, Tatsuumi Sasaki, MD, Shigeki Higashi, MD, ${ }^{a}$ Katsuhisa Onoguchi, MD, ${ }^{a}$ Kei Tanaka, MD, ${ }^{a}$ and Takahiro Shibata, MD, Saitama, Japan

The CarboMedics bileaflet prosthetic mechanical valve (CarboMedics. Inc., Austin, Tex.) has been used since 1986. Good results without any structural problems have been reported with this device. ${ }^{1}$ We present one case of mitral valve replacement with this valve that resulted in severe anemia caused by hemolysis 3 years after implantation. We believe that a leak occurred between the valve orifice and the titanium stiffening ring at the position of the hinge. This is the first published report of a complication of this type with this valve.

In February 1984 a 60-year-old man with New York Heart Association functional class III symptoms under-

From the Departments of Cardiovascular Surgery ${ }^{\mathrm{a}}$ and Cardiology, 'Saitama Ohara Cardiovascular Center, Saitama, Japan.

Accepted for publication April 19, 1995.

J Thorac Cardiovasc Surg 1996;111:277-8

Copyright (C) 1996 by Mosby-Year Book, Inc.

$0022-5223 / 96 \$ 5.00+0 \quad \mathbf{1 2 / 5 4 / 6 5 6 7 3}$ went mitral valve replacement with a $31 \mathrm{~mm}$ IonescuShiley pericardial valve (Shiley, Inc., Irvine, Calif.). Four years later, in February 1991, restricted movement of the valve caused by calcification was diagnosed. The patient was operated on again, and the mitral prosthesis was removed and replaced with a $29 \mathrm{~mm}$ CarboMedics bileaflet valve. The postoperative course was complicated by mediastinitis; however, the patient was discharged from the hospital in good condition. Follow-up occurred every 3 months; blood studies in December 1993 showed no anemia and a normal lactate dehydrogenase level. In April 1994, at the age of 70 years, the patient reported the recent onset of fatigue. At that time, transthoracic echocardiography demonstrated aortic valve stenosis with a pressure gradient of $60 \mathrm{~mm} \mathrm{Hg}$. The leakage at the mitral valve, however, was overlooked. In July 1994, the patient was hospitalized for exacerbation of his symptoms. Routine laboratory tests showed severe anemia (erythrocyte count $220 \times 10^{3} \mathrm{cells} / \mathrm{ml}$, hemoglobin $\left.7.8 \mathrm{~g} / \mathrm{dl}\right)$ and elevation of lactate dehydrogenase (2411 IU/L) and indirect bilirubin $(1.8 \mathrm{mg} / \mathrm{dl})$ levels. A perivalvular mitral leak

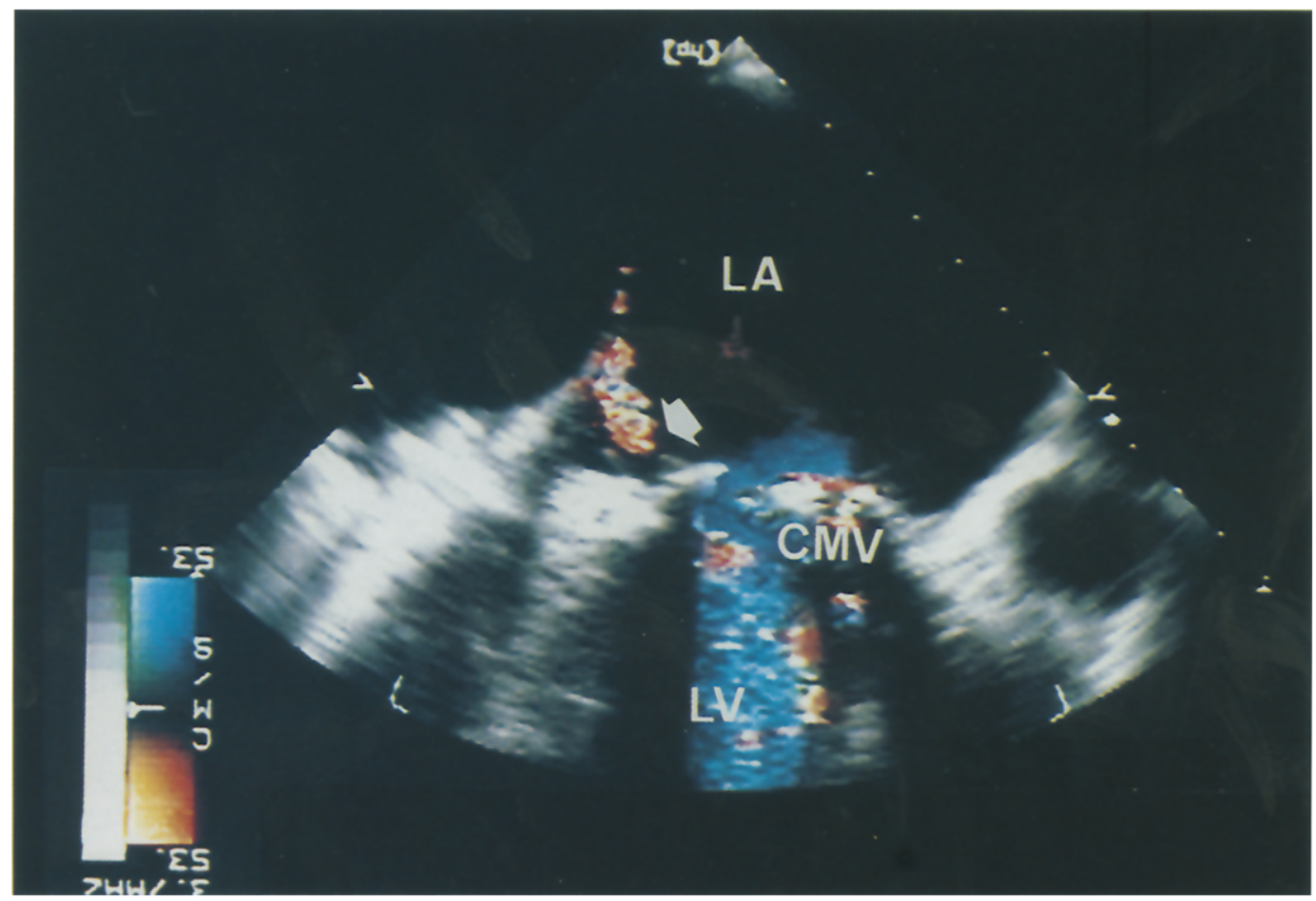

Fig. 1. Transesophageal echocardiography. $L A$, Left atrium; $L V$, left ventricle; $C M V$, CarboMedics valve. The white arrow indicates valvular leakage. 


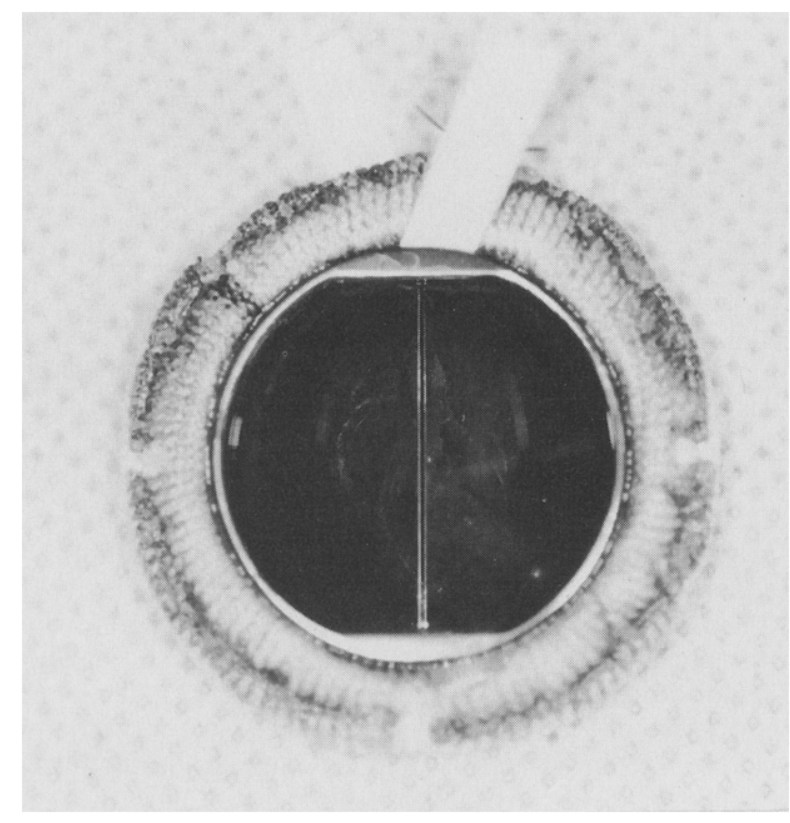

Fig. 2. Explanted CarboMedics $27 \mathrm{~mm}$ mitral valve. A paper strip has been inserted in the space between the prosthetic orifice and titanium stiffening ring (sewing cuff).

was diagnosed by means of transesophageal echocardiography (Fig. 1). The hemolysis gradually progressed, with a further decline in the hemoglobin concentration (5.9 $\mathrm{gm} / \mathrm{dl}$ ) and increase in the lactate dehydrogenase concentration $(5140 \mathrm{IU} / \mathrm{L})$.

The patient underwent a third operation in September 1994. There were extensive adhesions between the sternum and the aorta, and the right ventricle required considerable dissection before cardiopulmonary bypass could be initiated. The aortic valve was fused at the commissure between the right and noncuspid valves. Commissurotomy and decalcification of the thickened cusps restored cusp movement. Direct visual examination of the mitral prosthesis showed neither thrombi nor pannus overgrowth of the prosthesis ring. Examination showed no detachment of the sewing cuff from the mitral ring. There was no sign of infection in the heart. A Foley catheter was inserted through the aortic valve. The balloon was inflated to occlude the left ventricular outflow tract, and saline solution was injected into the left ventricle. No leakage was detected between the sewing cuff of the mitral prosthesis and the patient's mitral anulus. Regurgitation was detected between the sewing cuff and the prosthetic valve orifice at the hinge (Fig. 2). The valve was removed and a CarboMedics valve of the same size was implanted. Intractable postoperative bleeding (oozing), complicated by marked reduction of platelets, necessitated massive blood transfusion. The patient had disseminated intravascular coagulopathy after the operation. Multiorgan failure ensued, and he died 17 days after operation.

During the last 8 years, the CarboMedics heart valve has been implanted in patients from more than 50 countries. Last year, the U.S. Food and Drug Administration recommended market approval of this valve after 6 years of clinical investigation in the United States. Approximately 110,000 valves have been implanted, and the pyrolytic carbon components have proved satisfactory in terms of both durability and anticoagulation levels. ${ }^{1,2}$ The titanium stiffening ring is designed to protect the pivots and leaflets of the orifice and to reduce the risk of leaflet immobilization. The stiffening ring is mounted between the prosthetic orifice and the sewing cuff and secures it to the orifice ring. It allows in-site rotation. A trivial external hinge space is formed between the hinge and the titanium ring. The resulting leakage is not clinically significant because it is minimal and the space is occluded by clot formation immediately after implantation. It is not clear why in our patient external hinge leakage became apparent 3 years after operation and became clinically significant. The manufacturer has reported that, as a matter of routine product improvement, the external pyrolytic carbon coating of the orifice has been increased (personal communication). This has resulted in virtual elimination of the trivial clearance spaces.

Perivalvular leak is the most frequent nonstructural dysfunction with the CarboMedics valves as well as with others. Prosthetic valvular (external hinge) leak was not diagnosed by echocardiography. Differentiation of hinge leakage from perivalvular leak could not be determined. Careful attention toward the possible cause of external hinge leak between the sewing cuff and valvular orifice is essential when perivalvular leak is diagnosed.

\section{REFERENCES}

1. de Luca L, Vitale N, Gianolo B, Cafarella G, Piazza L, Cotrufo M. Mid-term follow-up after heart valve replacement with CarboMedics bileaflet prostheses. J Thorac Cardiovasc Surg 1993;106:1158-65.

2. Kratz JM, Crawford FA, Sade RM, Crumbley AJ, Stroud MR. St. Jude prosthesis for aortic and mitral valve replacement: a ten year experience. Ann Thorac Surg 1993;56:462-8. 